\title{
Review: health education interventions offering information plus sexual negotiation skill development promote increased condom use in women
}

\author{
Shepherd J, Weston R, Peersman G, et al. Interventions for encouraging sexual lifestyles and behaviours intended to prevent \\ cervical cancer. (Cochrane Review, latest version 25 May 1999.) In: Cochrane Library. Oxford: Update Software.
}

QUESTION: Do health education interventions promote sexual risk reduction behaviours associated with the transmission of human papillomavirus (HPV) in women?

\section{Data sources}

Studies were identified by searching the Cochrane Controlled Clinical Trials Register (1998, issue 4), Medline (1992-8), EMBASE/Excerpta Medica (1993-8), ERIC (1994-7), PsycLIT (1996-7), the Social Science Citation Index (1994-7), and handsearching selected journals and reference lists.

\section{Study selection}

Studies were selected if they evaluated educational interventions (eg, group or one-on-one learning and media campaigns) delivered by any provider (eg, teachers, health promotion experts, clinicians, and peer educators) in any setting (eg, schools, colleges, and clinics) among sexually and pre-sexually active women between 13 and 64 years of age and measured behavioural or clinical outcomes (eg, condom use and HPV or sexually transmitted disease $[\mathrm{STD}]$ incidence).

\section{Data extraction}

Study design, interventions, behavioural and clinical outcomes, participant characteristics, and methodological quality.

\section{Main results}

30 studies met the selection criteria. 10 studies (8 randomised controlled trials and 2 controlled trials without randomisation, mean follow up duration range 3-6 mo, 5089 women, age range 11-54 years, predominantly low socioeconomic status) were judged to be methodologically sound and were included in the analysis; all aimed to prevent HIV and other STDs (rather than cervical cancer) by providing factual information complemented with sexual negotiation skill development in women. Meta-analysis of studies was not done because of the heterogeneity in interventions, study populations, and outcome measures. In all 10 studies, health education interventions promoted sexual risk reduction behaviours, generally with self reported increases in the short term (typically $3 \mathrm{mo}$ ) use of condoms for vaginal intercourse. Health education information plus sexual negotiation skill development was associated with greater short term condom use than information alone (3 studies), no treatment (1 study), a waiting list control group (1 study), a single component intervention (1 study), or "generic" health promotion (2 studies).

\section{Conclusion}

Health education interventions that offer factual information complemented with sexual negotiation skill development promote short term increases in condom use for vaginal intercourse in women.

\section{COMMENTARY}

Limited information is available on the evaluation of interventions aimed at reducing HPV infection and preventing the development of cervical cancer. This systematic review by Shepherd $e t$ al evaluated the effectiveness of prevention programmes to reduce the transmission of HPV in women.

Studies that report significant results are more likely to be published than studies that report negative outcomes; however, unpublished studies might not differ from published studies in terms of methodological rigour, and their exclusion might bias the results of review papers. ${ }^{1}$ Although only published studies were evaluated in this review, various search methods were used. Criteria for study inclusion were clearly described and the methodological quality of the primary studies was assessed. To support the reproducibility and reduction of bias in the quality testing and data extraction process, 2 raters were used to review studies. The 10 methodologically sound studies that reported the effectiveness of condom use for short follow up periods had results similar to findings from a recent systematic review evaluating the effectiveness of primary prevention programmes to reduce STDs in adolescents. ${ }^{2}$

Most of the studies in this review were done on higher risk populations, compromising the ability to apply the results to a general population of women. Nurses working in public health settings or primary care areas where sexual health programmes are implemented should note that programmes showing a desirable effect were based on theory and included specific facts about STDs in combination with behavioural skills training, such as the correct use of latex condoms. We need more methodologically rigorous evaluations of carefully designed interventions that measure behavioural outcomes and biochemical markers for STD rates.

\section{Janet Yamada, RN, MSc Research Coordinator Hamilton-Wentworth Regional Social and Public Health Services Division \\ Hamilton, Ontario, Canada}

1 Dickersin K. The existence of publication bias and risk factors for its occurrence. JAMA 1990;263:1385-9.

2 Yamada J, DiCenso A, Feldman L, et al. A systematic review of the effectiveness of primary prevention programs to prevent sexually transmitted diseases in adolescents. Hamilton, Ontario: prepared by the effective public health practice project for the Ontario Ministry of Health, 1999
Source of funding: Cancer Research Campaign, UK.

For correspondence MrJ Shepherd, NCCHTA, Wessex Institute for Health Research and Development, University of Southampton, Bolderwood, Southampton, Hants SO16 7PX, UK. Fax $+44(0) 1703595639$. 